\title{
Causas das uveítes em serviço terciário em São Paulo, Brasil
}

\author{
Causes of uveitis in a tertiary center in São Paulo city, Brazil
}

\author{
Enéias Bezerra Gouveia ${ }^{1}$ \\ Joyce Hisae Yamamoto ${ }^{2}$ \\ Milena Abdalla ${ }^{3}$ \\ Carlos Eduardo Hirata ${ }^{4}$ \\ Paulo Kubo ${ }^{4}$ \\ Edilberto Olivalves $^{5}$
}

\section{RESUMO}

Objetivos: Analisar as causas das uveítes em pacientes atendidos no Serviço de Uveítes do HC-FMUSP no período de fevereiro a agosto de 2002. Métodos: Realizou-se estudo retrospectivo baseado em prontuários de 262 pacientes que compareceram no serviço no período acima. Os seguintes dados foram analisados: idade, sexo, diagnósticos anatômico e etiológico, atividade da doença e complicações oculares. Resultados: A idade média dos pacientes foi de 41 anos, sendo $60 \%$ do sexo feminino e $40 \%$ do masculino. O diagnóstico anatômico distribuiu-se em anterior (20\%), intermediário $(4,5 \%)$, posterior $(39,7 \%)$ e difuso $(31,3 \%)$. A etiologia foi determinada em $79,4 \%$ dos casos, sendo os diagnósticos mais freqüentes toxoplasmose (22\%), síndrome de Vogt-Koyanagi-Harada (13\%) e doença de Behçet (10\%). Causas infecciosas foram observadas em 79\% das uveítes posteriores, ao passo que causas não infecciosas em $61 \%$ das uveítes anteriores e $73 \%$ das difusas. Foram observados alguns diagnósticos raros, tais como uveíte associada ao HTLV-I/II, síndrome do anticorpo antifosfolípide e retinopatia auto-imune. Quantoà atividade de doença ocular, a doença estava ativa em $15 \%$ dos pacientes e inativa em $85 \%$. Ascomplicações mais comuns foram catarata (42\%) e glaucoma (13\%). Conclusões: Estudos epidemiológicos podem refletir diferentes padrões de etiologia das uveítes; no entanto eles podem mostrar características particulares de cada centro (terciário, critério de diagnóstico, interesses específicos). $\mathrm{O}$ presente estudo demonstra a importância das uveítes endógenas (síndrome de Vogt-Koyanagi-Harada e doença de Behçet), assim como das uveítes infecciosas (toxoplasmose e toxocaríase) em nosso meio.

Descritores: Uveíte/etiologia; Uveíte/epidemiologia; Estudo retrospectivo; Serviços de saúde comunitária

\section{INTRODUÇ̃̃̃O}

As uveítes, processo inflamatório intra-ocular comprometendo a úvea e estruturas vizinhas, são responsáveis por 5 a $20 \%$ das causas de cegueira na América do Norte ${ }^{(1)}$. Em nosso meio, uveíte é o principal diagnóstico encontrado em indivíduos que frequientam instituições para reabilitação visual $^{(2)}$ e é responsável por $4 \%$ dos atendimentos em serviço de urgên$\mathrm{cia}^{(3)}$. Sua incidência varia de 14 a $28 \mathrm{em} 100 \mathrm{mil}^{(4-5)}$.

Estudos epidemiológicos nas uveítes são importantes, visto que fatores demográficos tais como idade, sexo, raça, aspectos socioeconômicos, ocupação, hábitos de vida, origem geográfica, podem influenciar nos mesmos. A comparação de estudos realizados em diferentes regiões do mundo e em diferentes épocas permite estabelecer tendências e especular fatores relevantes para a etiopatogenia das uveítes. A literatura mundial relata grandes 
variações geográficas na etiologia das uveítes ${ }^{(6)}$. O presente trabalho tem como objetivo analisar a distribuição das uveítes em pacientes atendidos no Serviço de Uveítes da Clínica Oftalmológica do Hospital das Clínicas da Faculdade de Medicina da Universidade de São Paulo (HC-FMUSP), no período de fevereiro a agosto de 2002 .

\section{MÉTODOS}

Realizou-se estudo retrospectivo baseado em prontuários de pacientes que freqüentaram o Serviço de Uveítes da Clínica Oftalmológica do HC-FMUSP, no período de fevereiro a agosto de 2002, com o cuidado de inseri-los apenas uma vez na estatística. Os seguintes dados foram analisados: idade, sexo, diagnósticos anatômico e etiológico, atividade de doença e complicações oculares. Atividade de doença foi definida pela presença de hiperemia pericerática ou límbica associada à presença de células na câmara anterior, células no vítreo associadas ao edema cistóide de mácula, edema ou exsudação da retina ou edema e hemorragias perivasculares. As complicações oculares observadas foram: ceratopatia em faixa, sinéquias, seclusão pupilar, catarata, glaucoma, rotura de retina, descolamento de retina, hemorragia vítrea, edema macular cistóide, membrana neovascular sub-retiniana, fibrose de retina, hipotonia ocular e atrofia bulbar. Os pacientes incluídos neste estudo foram submetidos à rotina do Serviço de Uveítes, que consistiu em: exame ocular (medida da acuidade visual corrigida, exame ocular externo, biomicroscopia dos segmentos anterior e posterior, oftalmoscopia binocular indireta e tonometria de aplanação); exames complementares oculares, como ultrasom, angiofluoresceinografia da retina, campimetria visual, exames eletrofisiológicos da retina, quando necessários; e exames sistêmicos de acordo com o diagnóstico anatômico e/ ou etiológico ou das complicações observadas. Os critérios para diagnóstico das uveítes foram os estabelecidos pelo Grupo Internacional de Estudo das Uveítes ${ }^{(7)}$. Uveíte intermediária é, segundo esses critérios, aquela onde as manifestações inflamatórias se localizam principalmente na retina periférica, vítreo anterior e na região da pars plana. Nessa classificação, esse termo indica apenas a localização anatômica preferencial da uveíte, sem nenhuma indicação etiológica. Embora possa ser a expressão de uma série doenças como esclerose múltipla, doença de Behçet, sarcoidose, HTLV-I/II ("human T-lymphotropic virus types I and II"), sífilis, tuberculose, toxocaríase, entre outras, na maioria das vezes manifesta-se como uma uveíte isolada, cujos mecanismos etiopatogênicos não estão bem definidos, com provável componente auto-imune e que foi descrita na literatura com vários nomes como: pars planite, uveíte periférica, ciclite crônica e coriorretinite periférica e que nesse trabalho denominaremos de uveíte intermediária do tipo pars planite, independente da presença do "snow banks".

No período do levantamento, um protocolo específico com indivíduos portadores assintomáticos do HTLV-I/II estava sendo realizado, sendo incluído na análise deste estudo somente os indivíduos com alterações inflamatórias intra-oculares.

\section{RESULTADOS}

Foram analisadas 262 fichas de pacientes que freqüentaram o Serviço de Uveítes do HC-FMUSP no período de 6 meses definido pelo estudo. A idade dos pacientes na data do levantamento variou de 4 a 88 anos, com idade média de 41 anos, com a seguinte distribuição (Tabela 1): entre 0 e 16 anos, 25 pacientes (9\%), entre 17 e 40 anos, 108 pacientes $(41 \%)$ e maior que 40 anos, 129 pacientes (50\%), sendo 159 (60\%) mulheres e $103(40 \%)$ homens. Destes 262 pacientes, $25(9,5 \%)$ apresentavam seguimento menor que 1 ano e o restante seguimento maior que 12 meses. Quanto à classificação das uveítes segundo o diagnóstico anatômico (Quadro 1), 20\% eram uveíte anterior, 4,5\% uveíte intermediária, 39,7\% uveíte posterior e 31,3\% uveíte difusa. Outros diagnósticos anatômicos observados foram: esclerouveíte em 4\% (10 casos), ceratouveíte e neurite em um caso cada. As vasculites de retina foram incluídas nas uveítes posteriores.

O diagnóstico etiológico das uveítes pode ser determinado em 79,4\% dos casos, sendo as etiologias mais freqüentemente encontradas toxoplasmose ocular 22\% (57 casos), síndrome de Vogt-Koyanagi-Harada 13\% (33 casos) e doença de Behçet 10\% (26 casos) (Quadro 1).

Quanto à atividade de doença ocular, na data do levantamento, 40 pacientes (15\%) tinham doença ativa e 222 pacientes $(85 \%)$ estavam sem atividade de doença.

As complicações encontradas foram: catarata (111 pacientes, $42 \%$ ), glaucoma (35 pacientes, 13\%), sinéquias posteriores e/ou seclusão pupilar (30 pacientes, 11,4\%), descolamento de retina regmatogênico (17 pacientes, $6 \%$ ), atrofia bulbar (6 pacientes, $2 \%$ ), ceratopatia em faixa ( 5 pacientes, $2 \%$ ) e outras complicações como sinéquias anteriores, hemorragia vítrea, edema macular cistóide, membrana neovascular sub-retiniana, fibrose de retina e hipotonia ocular ( 9 pacientes, $3 \%$ ). Noventa e dois pacientes (35\%) não apresentavam nenhuma complicação.

A distribuição da etiologia das uveítes segundo o diagnóstico anatômico está representado no quadro 1 e segundo a faixa etária na tabela 1 . As causas infecciosas foram responsáveis por $79 \%$ das uveítes posteriores. As causas não infecciosas foram responsáveis por $61 \%$ das uveítes anteriores e $73 \%$ das uveítes difusas. Nas uveítes anteriores, destacaram-se como principais etiologias nas faixas etárias de 0-16 e de 17-40 anos, a artrite reumatóide juvenil, e na faixa etária acima de 40 anos a espondiloartropatia. Nas uveítes posteriores, a toxoplasmose predominou nos indivíduos acima de 16 anos e a toxocaríase naqueles com até 16 anos. Nas uveítes difusas, as etiologias mais freqüentes foram doença de Behçet e síndrome de Vogt-Koyanagi-Harada. Não se definiu o diagnóstico etiológico em $25 \%$ dos casos das uveítes anteriores, em 15,4\% das uveítes posteriores e em 19,5\% das uveítes difusas. 


\begin{tabular}{|c|c|c|}
\hline Faixa etária (anos) & Etiologia das uveítes & Número de casos \\
\hline \multirow[t]{6}{*}{$0-16$} & Sub-total & $25(9 \%)$ \\
\hline & Artrite reumatóide juvenil & 5 \\
\hline & Toxocaríase & 9 \\
\hline & Toxoplasmose & 3 \\
\hline & Pars planite & 3 \\
\hline & Idiopático & 5 \\
\hline \multirow[t]{12}{*}{$17-40$} & Sub-total & $108(41 \%)$ \\
\hline & Toxoplasmose & 35 \\
\hline & Doença de Behçet & 14 \\
\hline & Síndrome de Vogt-Koyanagi-Harada & 11 \\
\hline & Artrite reumatóide juvenil & 6 \\
\hline & Tuberculose & 6 \\
\hline & Espondilite anquilosante & 4 \\
\hline & Toxocaríase & 3 \\
\hline & Sarcoidose & 3 \\
\hline & Iridociclite heterocrômica de Fuchs & 2 \\
\hline & Outros* & 10 \\
\hline & Idiopático & 14 \\
\hline \multirow[t]{17}{*}{$>40$} & Sub-total & $129(50 \%)$ \\
\hline & Síndrome de Vogt-Koyanagi-Harada & 22 \\
\hline & Toxoplasmose & 19 \\
\hline & Doença de Behçet & 12 \\
\hline & Espondilite anquilosante & 6 \\
\hline & Tuberculose & 4 \\
\hline & Doença de Chrön & 3 \\
\hline & Sarcoidose & 3 \\
\hline & Iridociclite heterocrômica de Fuchs & 3 \\
\hline & HTLV-I/II & 3 \\
\hline & Artrite reumatóide do adulto & 2 \\
\hline & Sífilis & 2 \\
\hline & Coroidite multifocal & 2 \\
\hline & Oftalmia simpática & 2 \\
\hline & Pars planite & 3 \\
\hline & Outros* & 9 \\
\hline & Idiopático & 34 \\
\hline
\end{tabular}

\section{DISCUSSÃO}

Este estudo retrospectivo analisa a distribuição das uveítes em pacientes atendidos no Serviço de Uveítes da Clínica Oftalmológica do Hospital das Clínicas da Faculdade de Medicina da Universidade de São Paulo, no período de fevereiro a agosto de 2002. O quadro 2 apresenta um resumo de alguns estudos epidemiológicos das uveítes realizados em diferentes regiões do mundo, em diferentes épocas e em diferentes serviços, incluindo o resultado do presente trabalho. Estes estudos permitem estabelecer tendências e especular fatores relevantes para a etiopatogenia das uveítes de uma forma geral. Enquanto na década de 60, Woods et al. atribuíram $60 \%$ das uveítes à toxoplasmose e à tuberculose ${ }^{(6)}$, levantamentos nas décadas de 80 e 90 demonstram um aumento no diagnóstico de uveítes endógenas como a uveíte relacionada ao HLA-B27, vasculites de retina, sarcoidose e síndrome de Vogt-Koyanagi-Harada ${ }^{(8-12)}$. Já os levantamentos realizados no Japão ${ }^{(13)}$, onde predominam a doença de Behçet, sarcoidose e síndrome de Vogt-Koyanagi-Harada, apontam para diferenças étnicas na prevalência das uveítes. Em contrapartida, os levantamentos nacionais demonstram a importância da toxoplasmose no nosso meio, independente do período ${ }^{(5,8-9,14-17)}$. Estes mesmos levantamentos também apontam para a importância de causas infecciosas como tuberculose, sífilis, toxocaríase e hanseníase. Dentre as causas não infecciosas, no Brasil, destacam-se a doença de Behçet e síndrome de Vogt-Koyanagi-Harada ${ }^{(8-9,17)}$. Fatores inerentes a cada serviço que podem influenciar os resultados são vários: interesse de estudo por determinada doença, forma de encaminhamento dos doentes, critérios de 


\begin{tabular}{|c|c|c|c|c|c|c|}
\hline Etiologia & $\begin{array}{c}\text { Todas } \\
\text { as uveítes } \\
(262 ; 100 \%)^{\#}\end{array}$ & $\begin{array}{l}\text { Anterior } \\
(52 ; 20 \%)\end{array}$ & $\begin{array}{c}\text { Interm. } \\
(12 ; 4,5 \%)\end{array}$ & $\begin{array}{c}\text { Posterior } \\
(104 ; 40 \%)\end{array}$ & $\begin{array}{c}\text { Difusa } \\
(82 ; 31 \%)\end{array}$ & $\begin{array}{c}\text { Outras } \\
\text { manifest.* } \\
(12 ; 4,5 \%)\end{array}$ \\
\hline \multicolumn{7}{|l|}{ Infecciosa } \\
\hline Toxoplasmose & 57 & & & 57 & & \\
\hline Toxocaríase & 12 & & & 12 & & \\
\hline Tuberculose & 10 & & & 7 & 3 & \\
\hline HTLV-I/II & 4 & & 3 & & & 1 \\
\hline Sífilis & 3 & & & 2 & 1 & \\
\hline Retinite por citomegalovírus (HIV+) & 2 & & & 2 & & \\
\hline Coroidite por Pneumocistis carinii (HIV+) & 1 & & & 1 & & \\
\hline Ceratouveíte herpética & 1 & & & & & 1 \\
\hline Necrose aguda de retina & 1 & & & 1 & & \\
\hline Moléstia de Hansen & 1 & 1 & & & & \\
\hline Sub-total & 92 (35\%) & $1(2 \%)$ & $3(25 \%)$ & $82(79 \%)$ & $4(5 \%)$ & $2(17 \%)$ \\
\hline \multicolumn{7}{|l|}{ Não infecciosa } \\
\hline Síndrome de Vogt-Koyanagi-Harada & 33 & & & & 33 & \\
\hline Doença de Behçet & 26 & & & & 26 & \\
\hline Artrite reumatóide juvenil & 12 & 12 & & & & \\
\hline Espondilite anquilosante & 10 & 10 & & & & \\
\hline Sarcoidose & 6 & 4 & 1 & & 1 & \\
\hline Doença de Chrön & 3 & 3 & & & & \\
\hline Artrite reumatóide do adulto & 2 & 1 & & & & 1 \\
\hline Lupus eritematoso sistêmico & 2 & 1 & & 1 & & \\
\hline Síndrome antifosfolípide & 1 & & & 1 & & \\
\hline Psoríase & 1 & & 1 & & & \\
\hline Síndrome de Reiter & 1 & 1 & & & & \\
\hline Sub-total & 97 (37\%) & $32(61 \%)$ & $2(17 \%)$ & $2(2 \%)$ & $60(73 \%)$ & $1(8 \%)$ \\
\hline \multicolumn{7}{|l|}{ Doenças puramente oculares } \\
\hline Pars planite & 7 & & 7 & & & \\
\hline Iridociclite heterocrômica de Fuchs & 5 & 5 & & & & \\
\hline Oftalmia simpática & 2 & & & & 2 & \\
\hline Coroidite multifocal & 2 & & & 2 & & \\
\hline Síndrome de Possner Schlossman & 1 & 1 & & & & \\
\hline Coroidite serpiginosa & 1 & & & 1 & & \\
\hline Retinopatia auto-imune & 1 & & & 1 & & \\
\hline Sub-total & $19(7 \%)$ & $6(11 \%)$ & $7(58 \%)$ & $4(4 \%)$ & $2(2 \%)$ & 0 \\
\hline Idiopática & 54 (21\%) & $13(25 \%)$ & 0 & $16(15 \%)$ & $16(19 \%)$ & $9(75 \%)$ \\
\hline
\end{tabular}

diagnóstico, acesso a métodos diagnósticos especializados. Estudos prospectivos incluindo pacientes atendidos em serviço de urgência e que tenham um seguimento mínimo de 6 a 12 meses, permitindo a observação do curso da uveíte, a investigação clínica e laboratorial, poderão demonstrar de forma mais precisa as tendências reais de incidência de cada uveíte. $\mathrm{O}$ presente estudo, realizado de forma retrospectiva, incluindo todos os pacientes que foram atendidos no período, supervalorizou a freqüência de algumas doenças mais graves como síndrome de Vogt-Koyanagi-Harada, e doença de Behçet, pois esses pacientes, embora incluídos no estudo uma única vez, são reavaliados de modo mais freqüente, aumentando a chance da sua inclusão no período do estudo.

Independente dos fatores mencionados acima, o presente levantamento permitiu reforçar a importância de algumas doenças. Nas uveítes anteriores, destacaram-se como principais etiologias a artrite reumatóide juvenil, na faixa etária abaixo de 40 anos, e a espondiloartropatia, na faixa etária acima de 40 anos. A alta prevalência de artrite reumatóide juvenil em adultos entre 17 e 40 anos deve-se à inclusão no estudo da idade atual e não da idade na qual a doença se instalou. Na artrite reumatóide juvenil, principalmente na forma pauci-articular, a uveíte anterior é crônica, freqüentemente bilateral, com ausência de sinais como hiperemia conjuntival, resultando num diagnóstico tardio e com a presença de seqüelas que comprometem a função visual ${ }^{(18)}$. Já nas espondiloartropatias, a uveíte anterior é aguda, recorrente, bilateral, porém assimétrica e raramente simultânea. A espondilite anquilosante, o protótipo 


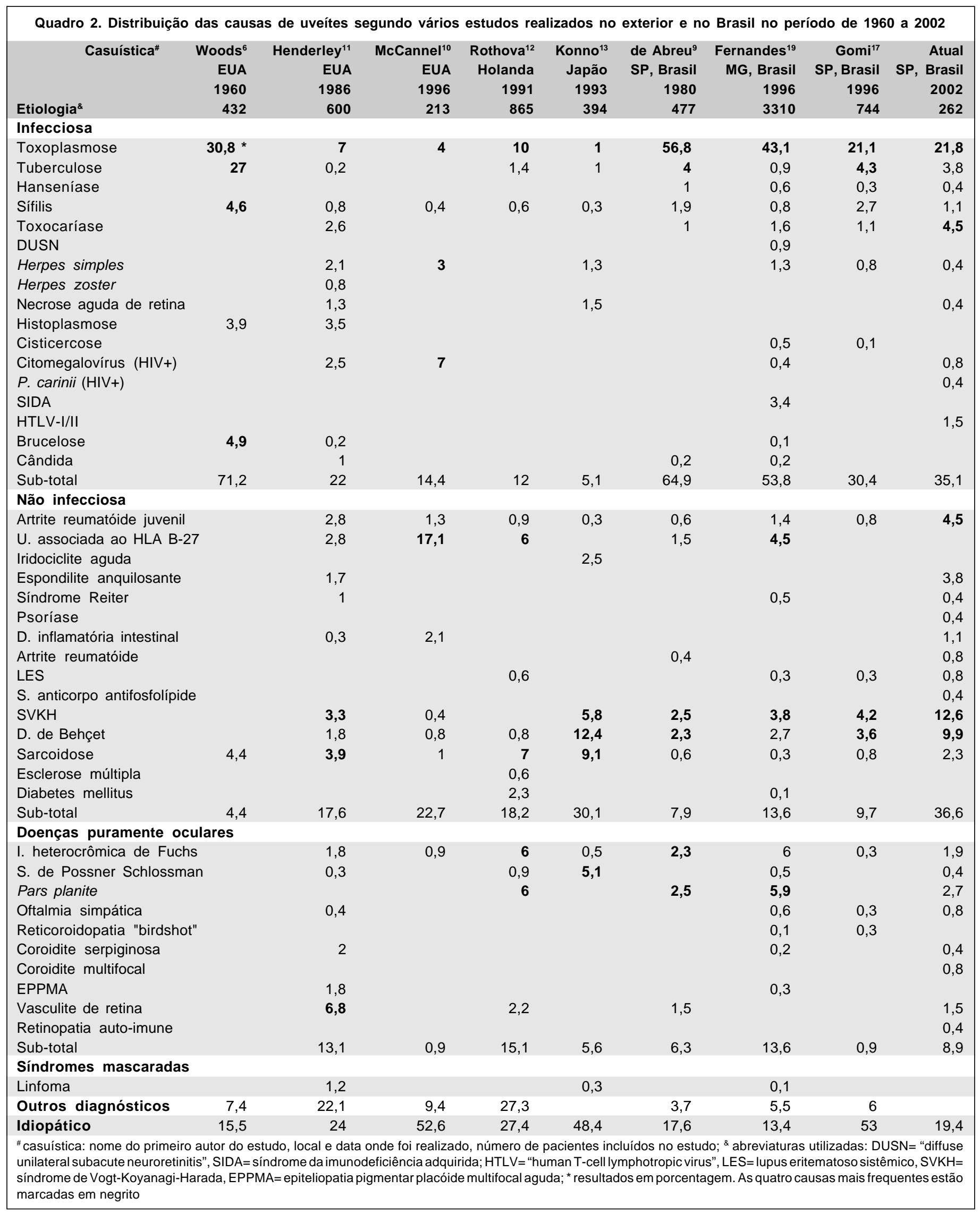


das espondiloartropatias, começa, habitualmente, na segunda ou terceira década, acometendo 3 vezes mais os homens do que as mulheres. A uveíte anterior aguda ocorre em cerca de $20 \%$ dos pacientes com espondilite anquilosante, sendo a manifestação extra-articular mais comum, podendo preceder em anos os sintomas clínicos da artrite $^{(19)}$.

A toxoplasmose foi a causa mais comum de uveíte em pacientes acima de 17 anos. Quer pela frequiência, quer pela gravidade, a prevenção e o tratamento adequado da toxoplasmose ocular assumem extrema importância para se evitar a cegueira em nosso meio. Na infância, a toxocaríase foi a principal causa de uveíte posterior. O seu diagnóstico baseia-se essencialmente no quadro clínico substanciado pelos antecedentes epidemiológicos e pela presença de anticorpos anti-Toxocara canis no soro. A pesquisa de anticorpos específicos no humor aquoso ou no vítreo pode também contribuir para o diagnóstico da toxocaríase ocular. Devemos lembrar das 3 formas clínicas clássicas, granuloma no pólo posterior, granuloma periférico e endoftalmite ${ }^{(20)}$. Embora a maioria dos estudos epidemiológicos realizados no Brasil ressalte a importância da toxoplasmose em qualquer faixa etária ${ }^{(9,17,19)}$, a estreita colaboração que o Serviço de Uveítes mantém com o laboratório de Imu-nologia e Soroepidemiologia do Instituto de Medicina Tropical de São Paulo da FMUSP, laboratório de referência para pesquisa de anticorpos anti- $T$. canis no soro e nos fluidos intra-oculares, pode ter influenciado os nossos resultados.

Outros aspectos interessantes podem ser observados através do presente estudo. A porcentagem de uveítes sem diagnóstico etiológico $(20,6 \%)$ é comparável às demais casuísticas e é relativamente mais baixa à obtida no levantamento em 1996 $(53 \%)$ no nosso serviço ${ }^{(17)}$. Alguns fatores contribuíram para esta queda. Dentre os 262 pacientes incluídos, a maioria eram pacientes com seguimento maior do que 1 ano, permitindo um diagnóstico etiológico de modo mais preciso. A reavaliação do prontuário e do paciente no retorno durante o período limitado por este estudo também foi fundamental para este resultado. Outra observação interessante foi que dentre os 12 pacientes com uveíte intermediária, 3 apresentavam infecção pelo HTLV-I/II, sugerindo uma forte relação com a doença. O HTLV-I/II é um retrovírus transmitido principalmente através do aleitamento materno, relação sexual e elementos celulares do sangue. O Brasil é considerado uma região endêmica para este retrovírus, sendo que as suas principais manifestações clínicas são a leucemia ou linfoma de célula T do adulto, paraparesia espástica tropical e a uveíte. Dentre as várias formas de uveíte, a mais comum é a uveíte intermediária ${ }^{(21)}$. A soroprevalência para o HTLV-I/II, em nosso meio, em pacientes com uveíte de etiologia indeterminada varia de $1,8 \%$ a $3,6 \%$, e em doadores de sangue é de $0,32 \%$, sugerindo que a infecção por este retrovírus é dez vezes maior em pacientes com uveíte do que em doadores de sangue ${ }^{(22)}$. Desta forma, o HTLV-I/II deve ser lembrado como um possível agente etiológico de uveíte. E, por último, algumas doenças bastante incomuns, tais como a síndrome do anticorpo antifosfolípide e a retinopatia autoimune, foram relembradas. A primeira é mais freqüentemente acompanhada nos serviços de retina devido aos fenômenos oclusivos de retina. É caracterizada por eventos trombóticos arteriais e venosos recorrentes na presença de anticoagulante lúpico e/ou teste de anticardiolipina positivo ${ }^{(8)}$. Já a retinopatia auto-imune é bastante rara. É caracterizada por alterações degenerativas de retina e presença de anticorpos antiproteínas presentes no fotorreceptor. Geralmente é uma manifestação paraneoplásica de um carcinoma de pulmão ou de outra localidade ${ }^{(23)}$, porém tem sido descritos casos na ausência de neoplasia $^{(24)}$.

\section{CONCLUSÃO}

O presente estudo retrospectivo analisou a distribuição das uveítes num serviço terciário permitindo observar a alta prevalência da toxoplasmose, de uveítes endógenas (Síndrome de Vogt-Koyanagi-Harada e doença de Behçet) e da toxocaríase em nosso serviço. Revelou também que a associação de uveíte intermediária e infecção por HTLV-I/II pode ser muito importante. Síndrome do anticorpo antifosfolípide e retinopatia auto-imune devem ser lembrados em situações específicas.

O conhecimento de novas entidades clínicas e o melhor diagnóstico das doenças já conhecidas tem permitido o melhor esclarecimento da etiologia das uveítes. No entanto, o diagnóstico definitivo ainda não é possível em um quinto delas, estimulando a contínua pesquisa de novos agentes e de novas entidades clínicas.

O presente estudo, assim como a grande maioria dos estudos epidemiológicos de uveíte, é proveniente de centros terciários de saúde, podendo trazer erros e tendências não reais na distribuição das uveítes na população geral ${ }^{(10)}$. Ainda assim a relativa freqüência com a qual várias formas de uveítes são observadas em serviço terciário de referência reflete a comunidade na qual o centro está localizado.

$\mathrm{O}$ resultado destes e de futuros estudos de uveítes podem ajudar a identificar o perfil epidemiológico das uveítes no Brasil, sendo isto fundamental para o estabelecimento das nossas prioridades junto ao ensino, ao atendimento da população e à pesquisa oftalmológica.

\section{ABSTRACT}

Purpose: To analyze retrospectively the diagnosis of patients being followed up at the Uveitis Service of Hospital das Clínicas, University of São Paulo Medical School, during a 6month period, from February to August, 2002. Methods: 262 patients, who attended the Uveitis Service during that period, had their charts reviewed and their diagnoses confirmed during the appointment. The following data were analyzed: age, gender, anatomic and etiological diagnoses, disease activity and ocular complications. Results: Patients' mean age was 41 years, $60 \%$ being female and $40 \%$ male. Distribution according to anatomic diagnosis was: anterior $20 \%$, intermediate $4.5 \%$, 
posterior $39.7 \%$ and diffuse $31.3 \%$. Uveitis etiology was determined in $80 \%$ of the cases, and the most common causes were toxoplasmosis $22 \%$, Vogt-Koyanagi-Harada syndrome $13 \%$ and Behçet's disease $10 \%$. Infectious causes were present in $79 \%$ of posterior uveitis, whereas non-infectious systemic diseases were observed in $61 \%$ of anterior and $73 \%$ of diffuse uveitis. Uncommon diagnoses were observed such as HTLV-I/II related-uveitis, antiphospholipid syndrome and autoimmune retinopathy. Concerning disease activity, $15 \%$ were active and $85 \%$ were inactive. The most frequent ocular complications were cataract $(42 \%)$ and glaucoma (13\%). Conclusions: Epidemiological studies may demonstrate different patterns of uveitis of etiology; nevertheless they also reflect the particular characteristics of each center (tertiary, diagnosis criteria, special interests). The present study demonstrates in our midst the importance of endogenous uveitis such as VogtKoyanagi-Harada syndrome and Behçet's disease, as well as of infectious uveitis such as toxoplasmosis and toxocariasis.

Keywords: Uveitis/etiology; Uveitis/epidemiology; Retrospective studies; Community health services

\section{AGRADECIMENTOS}

Sra. Elisa Lieberman pela assessoria no atendimento aos pacientes com uveíte.

\section{REFERÊNCIAS}

1. Kahn HA, Moorhead HB. Statistics on blindness in the model reporting area; 1969-70. US Department of Health, Education and Welfare Publication NIH 1973. p.73-427.

2. Kara-José N, Carvalho KMM, Pereira VL, Venturini NHB, Gasparetto MEFR, Guchiken MT. Estudo retrospectivo dos primeiros 140 casos atendidos na clínica de visão sub-normal do Hospital das Clínicas da Unicamp. Arq Bras Oftalmol 1988; 51:65-9.

3. Nascimento VP, Takei LM, Takahashi WY, Kara-José N. Epidemiologia dos atendimentos de seis meses do pronto socorro oftalmológico do HCFMUSP [resumo]. [Apresentado no $30^{\circ}$ Congresso Brasileiro de Oftalmologia; 1999; Recife].

4. Guex-Crosier Y. Epidemiology of uveitis. Rev Prat 1999; 49: 1989-94.
5. Gehlen ML, Dabul VM, Obara SS, Grebos SP, Moreira CA. Incidência e etiologia de uveítes em Curitiba. Arq Bras Oftalmol 1999; 62:622-6.

6. Woods AC. Modern concepts of the etiology of uveitis. Am J Ophthalmol 1960; 50:1170-87.

7. Bloch-Michel E, Nussenblatt RB. International Uveitis Study Group recommendations for the evaluation of intraocular inflammatory disease. Am J Ophthalmol 1987; 103:234-5.

8. Oréfice F. Uveíte clínica e cirúrgica. São Paulo: Contexto; 2000.

9. Abreu MT, Hirata PS, Belfort Jr. R, Neto SD. Uveítes em São Paulo. Estudo epidemiológico, clínico e terapêutico. Arq Bras Oftalmol 1980; 43:10-6.

10. McCannel CA, Holland GN, Helm CJ, Cornell PJ, Winston JV, Rimmer TG. Causes of uveitis in the general practice of ophthalmology. UCLA Community-Based Uveitis Study Group. Am J Ophthalmol 1996; 121:35-46.

11. Henderly DE, Genstler AJ, Smith RE, Rao NA. Changing patterns of uveitis. Am J Ophthalmol 1987; 103:131-6.

12. Rothova A, Buitenhuis HJ, Meenken C, Brinkman CJJ, Linssen A, Alberts C, Luyendijk L, Kijlstra A. Uveitis and systemic disease. Br J Ophthalmol 1992; 76:137-41.

13. Konno Y, Numaga J, Fujino Y, Joukou S, Masuda K. Incidence of uveitis at Tokyo University Hospital. Jpn J Clin Ophthalmol 1993; 47:1243-7.

14. Albino GC, da Rocha AW, Jr Grumann A. perfil epidemiológico das uveítes na Grande Florianópolis [resumo]. Arq Bras Oftalmol 1997; 60:355-7. [Apresentado no $29^{\circ}$ Congresso Brasileiro de Oftalmologia; 1997; Goiânia - GO].

15. Garcia CAA, Gomes AHB, Barbosa MFA, Rocha MLR, Uchôa RAC. Aspectos clínicos e epidemiológicos das uveítes em Natal-RN. Rev Bras Oftalmol 2002; 61:121-9.

16. Argentino A, Pezzutti S, Sibinelli MA, Neufeuld CR. Análise de 1580 casos de uveítes da Santa Casa de São Paulo [resumo]. Arq Bras Oftalmol 1998; 61: 439.

17. Gomi CF, Makdissi FF, Yamamoto JH, Olivalves E. Estudo epidemiológico das uveítes. Rev Med (São Paulo) 1997; 76:101-8.

18. Damico FM, Hirata CE, Santoro SHW, Yamamoto JH, Kiss MHB, Olivalves E. Estudo da uveíte na artrite reumatóide juvenil. Arq Bras Oftalmol 1998; 61:695-9.

19. Fernandes LC, Oréfice F. Aspectos clínicos e epidemiológicos das uveítes em serviços de referência em Belo Horizonte, de 1970 a 1993. Parte I. Rev Bras Oftalmol 1996; 55:569-78.

20. Castro EFS, Yamamoto JH, Hirata CE, Yamashiro-Kanashiro EH, Peres BA. Diagnóstico da toxocaríase ocular- estudo retrospectivo. [resumo]. [Apresentado no $30^{\circ}$ Congresso Brasileiro de Oftalmologia; 1999; Recife,].

21. Yamamoto JH. Uveíte associada ao HTLV-I. In: Veronesi R, ed. Focaccia R, ed. Tratado de Infectologia. São Paulo: Atheneu, 1996.p.421-2.

22. Yamamoto JH, Segurado AA, Hirata CE, Sampaio MW, Souza EC, Nukui Y, Cliquet M, Saéz-Alquézar A, Olivalves E, Mochizuki M. Human T-cell lymphotropic vírus type I infection and ocular manifestations in São Paulo, Brazil. Arch Ophthalmol 1999; 117:513-7.

23. Thirkill CE, Roth AM, Keltner JL. Cancer-associated retinopathy. Arch Ophthalmol 1987; 105:372-5.

24. Mizener JB, Kimura AE, Adamus G, Thirkill CE, Goeken JA, Kardon RH. Autoimmune retinopathy in the absence of cancer. Am J Ophthalmol 1997; 123:607-18.

\title{
ABO ELETRÔNICO
}

\author{
Novo site
}

\section{Acesso: http://www.abonet.com.br}

